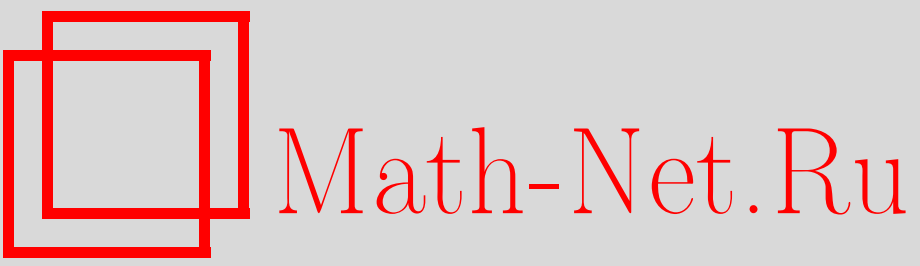

Н. А. Славнов, Интегральные уравнения для корреляционных функций квантового одномерного бозе-газа, ТМФ, 1999, том 121, номер 1, 117-138

DOI: https://doi.org/10.4213/tmf801

Использование Общероссийского математического портала Math-Net.Ru подразумевает, что вы прочитали и согласны с пользовательским соглашением

http://www.mathnet.ru/rus/agreement

Параметры загрузки:

IP : 18.209 .158 .208

26 апреля 2023 г., 10:33:23

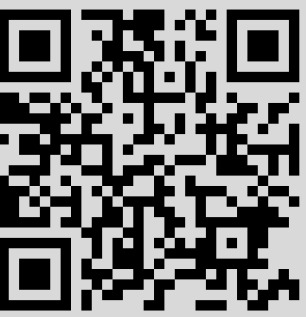




\section{ИНТЕГРАЛЬНЫЕ УРАВНЕНИЯ ДЛЯ КОРРЕЛЯЦИОННЫХ ФУНКЦИЙ КВАНТОВОГО ОДНОМЕРНОГО БОЗЕ-ГАЗА ${ }^{1)}$}

Рассматривается асимптотическое поведение температурных корреляционных функций квантового одномерного бозе-газа при больших расстояниях и временах. Получены интегральные уравнения, решения которых описывают данную асимптотику. Эти уравнения тесно связаны с уравнениями термодинамического анзаца Бете. В низкотемпературном пределе решения этих уравнений даны в терминах наблюдаемых модели.

\section{1. ВВЕДЕНИЕ}

Настояшая работа является продолжением ряда работ [1-5], посвяшенных изучению корреляционных функций квантового одномерного бозе-газа с дельтообразным взаимодействием. Здесь мы рассматриваем вопросы, связанные с асимптотикой температурных корреляционных функций при больших расстояниях и временах. Основным результатом работы является система интегральных уравнений (см. раздел 4), решения которой описывают данную асимптотику. Эти уравнения близки по форме к уравнениям термодинамического анзаца Бете, и, по-видимому, их решения тесно связаны с наблюдаемыми модели одномерного бозе-газа. Во всяком случае, в низкотемпературном пределе такая связь действительно существует.

Основным объектом нашего исследования является температурная корреляционная функция локальных полей

$$
\left\langle\Psi(0,0) \Psi^{\dagger}(x, t)\right\rangle_{T}=\frac{\operatorname{tr}\left(e^{-\frac{H}{T}} \Psi(0,0) \Psi^{\dagger}(x, t)\right)}{\operatorname{tr} e^{-\frac{H}{T}}} .
$$

Здесь $T$ - температура, $H$ - гамильтониан,

$$
H=\int d x\left(\partial_{x} \Psi^{\dagger}(x) \partial_{x} \Psi(x)+c \Psi^{\dagger}(x) \Psi^{\dagger}(x) \Psi(x) \Psi(x)-h \Psi^{\dagger}(x) \Psi(x)\right),
$$

\footnotetext{
1) Статья написана по заказу Редколлегии.
}

* Математический институт им. В. А. Стеклова РАН, Москва, Россия 
где $c>0$ - константа связи, $h$ - химический потенциал. Операторы $\Psi(x, t)$ и $\Psi^{\dagger}(x, t)$ являются каноническими бозе-полями:

$$
\left[\Psi(x, t), \Psi^{\dagger}(y, t)\right]=\delta(x-y)
$$

Уравнение движения, соответствуюшее гамильтониану (1.2), называется квантовым нелинейным уравнением Шредингера (НУШ).

Для удобства читателя приведем основные уравнения для наблюдаемых одномерного бозе-газа при конечной температуре. Энергия элементарного возбуждения (частицы) удовлетворяет уравнению Янга-Янга [6]

$$
\varepsilon(\lambda)=\lambda^{2}-h-\frac{T}{2 \pi} \int_{-\infty}^{\infty} K(\lambda, \mu) \ln \left(1+e^{-\frac{\varepsilon(\mu)}{T}}\right) d \mu, \quad K(\lambda, \mu)=\frac{2 c}{c^{2}+(\lambda-\mu)^{2}}
$$

При положительном химическом потенциале функция $\varepsilon(\lambda)$ имеет два вешественных корня $\varepsilon\left( \pm q_{T}\right)=0$. Полная спектральная плотность вакансий в газе задается уравнением

$$
2 \pi \rho_{t}(\lambda)=1+\int_{-\infty}^{\infty} K(\lambda, \mu) \vartheta(\mu) \rho_{t}(\mu) d \mu
$$

где функция

$$
\vartheta(\lambda)=\left(1+\exp \left[\frac{\varepsilon(\lambda)}{T}\right]\right)^{-1}
$$

играет роль фермиевского веса.

Импульс частицы, как функция спектрального параметра, тоже может быть найден из соответствуюшего интегрального уравнения. Однако в дальнейшем нам потребуется только производная импульса по спектральному параметру. Эта величина с точностью до коэффициента совпадает с полной плотностью вакансий:

$$
\frac{\partial k(\lambda)}{\partial \lambda}=2 \pi \rho_{t}(\lambda)
$$

Скорость частицы $v(\lambda)$ есть

$$
v(\lambda)=\frac{\partial \varepsilon}{\partial k}=\frac{\varepsilon^{\prime}(\lambda)}{k^{\prime}(\lambda)}=\frac{\varepsilon^{\prime}(\lambda)}{2 \pi \rho_{t}(\lambda)}
$$

Наконец, приведем также интегральное уравнение для фазы рассеяния:

$$
2 \pi F(\lambda, \nu)=\int_{-\infty}^{\infty} K(\lambda, \mu) \vartheta(\mu) F(\mu, \nu) d \mu+i \ln \left(\frac{i c+\lambda-\nu}{i c+\nu-\lambda}\right)
$$

Здесь и в дальнейшем ветвь логарифма выбирается так, чтобы $-\pi<\arg \ln z \leqslant \pi$. Beличина $S(\lambda, \nu)=\exp \{2 \pi i F(\lambda, \nu)\}$ является матрицей рассеяния двух частиц с импульсами $k(\lambda)$ и $k(\nu)$. 
Все приведенные термодинамические наблюдаемые потребуются нам в дальнейшем при описании асимптотики коррелящионной функции.

Рассмотрим поведение корреляционной функции (1.1) при $x \rightarrow \infty, t \rightarrow \infty$ и фиксированном отношении $\lambda_{0}=x / 2 t$. Одномерный бозе-газ является безмассовой моделью, поэтому при нулевой температуре коррелятор (1.1) убывает степенным образом:

$$
\left\langle\Psi(0,0) \Psi^{\dagger}(x, t)\right\rangle_{0} \longrightarrow C t^{-\Delta_{0}} .
$$

Показатель $\Delta_{0}$ может быть вычислен в рамках конформной теории поля [7]. При конечной температуре асимптотика меняется на экспоненциальную

$$
\left\langle\Psi(0,0) \Psi^{\dagger}(x, t)\right\rangle_{T} \longrightarrow C t^{-\Delta} e^{-\frac{t}{r}}
$$

Другими словами, нулевая температура является точкой фазового перехода. При конечной температуре методы конформной теории поля могут применяться лишь приближенно, например для вычисления низкотемпературных вкладов в корреляционньй радиус [7].

Метод детерминантного представления, применявшийся в работах $[1-5]$, в принципе позволяет рассматривать корреляционные функции при любой температуре. Особенно полезным данный метод оказывается при изучении асимптотик. В настоящей работе внимание сконцентрировано на главном, экспоненциальном законе убывания в формуле (1.10). Мы не будем интересоваться ни предэкспоненциальным фактором $t^{-\Delta}$, ни постоянным коэффициентом $C$ в (1.10). Иными словами, нашей целью является вычисление коррелящионного радиуса.

Содержание работы следующее. Раздел 2 носит обзорный характер. В нем мы даем краткое описание метода, основанного на представлении корреляционных функций в виде детерминантов Фредгольма, а также обсуждаем вопросы, связанные с конструкцией вспомогательных квантовых операторов - дуальных полей. В частности, мы объясняем причины появления этих операторов и их роль в вычислении корреляционных функций. Подробности можно найти в упоминавшихся выше работах [1-5]. В разделе 3 развивается техника усреднения операторов, зависящих от дуальных полей. Определено отображение усреднения, сопоставляющее квантовым операторам некоторые классические функции. Данное отображение позволяет вычислить асимптотику корреляционной функции (1.1). Это сделано в разделе 4, где приведена система интегральных уравнений, описывающих асимптотику. В пятом разделе рассмотрены некоторые конкретные случаи корреляционной функции (1.1). В частности, доказано, что в низкотемпературном приближении наши результаты в точности совпадают с результатами [7].

\section{2. ДУАЛЬНЫЕ ПОЛЯ}

Метод вычисления корреляционных функций с помощью фредгольмовых детерминантов подробно описан, например, в книге [8]. На первом этапе данного метода необходимо представить корреляционную функцию в виде определителя некоторого интегрального оператора, ядро которого зависит от расстояния и времени (и других физических параметров модели). Для корреляционной функции (1.1) такое представление 
было найдено в [1]. На втором этапе исследование полученного фредгольмова детерминанта может быть проведено с помощью методов теории классических точно решаемых уравнений. Дело в том, что упомянутые детерминанты оказываются $\tau$-функциями классических интегрируемых систем. В частности, для модели квантового одномерного бозе-газа они выражаются через решения классических разновидностей НУШ (в простейшем случае двухточечной корреляционной функции полей в пределе свободных фермионов соответствующее дифференциальное уравнение оказывается скалярным НУШ). Решения классических интегрируемых уравнений, в свою очередь, могут быть найдены с помошью методов задачи Римана. В частности, для нахождения асимптотик решений на больших временах и расстояниях используется нелинейный метод перевала [9], в принципе позволяющий получить полное асимптотическое разложение для корреляционной функции. Таким образом, вычисление асимптотик корреляторов в рамках описанного метода состоит из двух этапов:

1) представление коррелятора в виде детерминанта Фредгольма;

2) вычисление асимптотики полученного детерминанта с помощью методов задачи Римана и классических точно решаемых уравнений.

Однако так дело обстоит только в моделях, эквивалентных свободным фермионам (в квантовом НУШ - это предел бесконечной константы связи: $c \rightarrow \infty$ ). Вне предела свободных фермионов ситуация более сложная, и к описанной выше схеме необходимо добавить еше один шаг:

3) усреднение по вспомогательному вакууму.

Настоящая работа посвящена как раз этой последней стадии вычислений, поэтому мы остановимся на данном вопросе более подробно.

Для того чтобы получить детерминантное представление для корреляционных функций моделей несвободных фермионов (конечная константа связи в случае одномерного бозе-газа), необходимо вводить вспомогательные квантовые операторы - дуальные поля Корепина [10]. Такая необходимость вызвана наличием нетривиальной $S$-матрицы. Рассмотрим вкратце основную идею. Для вычисления корреляционной функции некоторого оператора $\mathcal{O}$ по состоянию $|a\rangle$ можно воспользоваться разложением по формфакторам

$$
\langle a|\mathcal{O}(x, t) \mathcal{O}(0,0)| a\rangle=\sum_{|b\rangle}|\langle a|\mathcal{O}(0,0)| b\rangle|^{2} e^{D_{a b}(x, t)} .
$$

Здесь состояния $|b\rangle$ пробегают полный набор, функция $D_{a b}(x, t)$ описывает закон дисперсии для данной модели. Величина $\langle a|\mathcal{O}(0,0)| b\rangle$ называется формфактором оператора $\mathcal{O}$, и, например, в квантовом НУШ она может быть явно вычислена с помощью алгебраического анзаца Бете. Так, для $\mathcal{O}=\Psi$ формфактор оказывается пропорциональным некоторому детерминанту:

$$
\langle a|\Psi(0,0)| b\rangle \sim \operatorname{det} M
$$

Подчеркнем, что, в отличие от моделей квантовой теории поля, здесь формфакторы строятся не над физическим вакуумом, а над голым фоковским вакуумом. Если состояния $|a\rangle$ и $|b\rangle$ параметризуются спектральными параметрами $\{\lambda\}$ и $\{\mu\}$, соответственно, 
то матричные элементы $M_{j k}$ являются функциями этих спектральных параметров

$$
M_{j k}=M_{j k}(\{\lambda\},\{\mu\})
$$

Исключительно важным оказывается характер зависимости $M_{j k}$ от спектральных параметров. Фактически, чтобы получить детерминантное представление для среднего (2.1), мы должны свести бесконечную сумму детерминантов в правой части (2.1) к единственному детерминанту. Это можно сделать только для специального вида матриц $M$, а именно, если $M_{j k}=M\left(\lambda_{j}, \mu_{k}\right)$, т.е. матричные элементы параметризуются одной функцией двух переменных $M(x, y)$, взятой в точках $x=\lambda_{j}$ и $y=\mu_{k}$. Это так называемые локальные матришы. В действительности элементы матрицы $M_{j k}$, описываюшей формфактор (2.2), содержат произведения двухчастичных $S$-матриц

$$
S(\lambda, \mu)=-\frac{i c+\lambda-\mu}{i c+\mu-\lambda}
$$

по всем параметрам $\{\lambda\}$ и $\{\mu\}$. Это приводит к тому, что для взаимодействуюших фермионов элементы $M_{j k}$ зависят сразу от всех спектральных параметров $M_{j k}=$ $M(\{\lambda\},\{\mu\})$ (нелокальные матрицы). В результате сведение бесконечной суммы детерминантов (2.1) к единственному детерминанту становится невозможным. С другой стороны, в пределе свободных фермионов $(c \rightarrow \infty) \quad S$-матрица становится тривиальной, и, как следствие, в функции $M$ остается зависимость только от двух переменных $M_{j k}=M\left(\lambda_{j}, \mu_{k}\right)$. Подробности и явные формулы можно найти в [1].

Введение дуальных полей исправляет ситуацию. Для приведения матрицы $M$ к локальному виду оказывается достаточно факторизовать $S$-матрищу

$$
S(\lambda, \mu)=f_{1}(\lambda) f_{2}(\mu),
$$

где $f_{1,2}$ - некоторые функции. Разумеется, для $S$-матришы (2.3) такая факторизация невозможна, если $f_{1,2}-$ классические функции. Однако этого легко достичь при помощи квантовых объектов.

Рассмотрим два оператора рождения $q_{\psi}(\lambda)$ и $q_{\phi}(\lambda)$, а также два оператора уничтожения $p_{\psi}(\lambda), p_{\phi}(\lambda)$, действуюшие во вспомогательном фоковском пространстве следующим образом:

$$
\left(0\left|q_{\psi}(\lambda)=\left(0\left|q_{\phi}(\lambda)=0, \quad p_{\psi}(\lambda)\right| 0\right)=p_{\phi}(\lambda)\right| 0\right)=0 .
$$

Ненулевые коммутационные соотношения имеют вид

$$
\left[p_{\phi}(\lambda), q_{\psi}(\mu)\right]=-\left[p_{\psi}(\lambda), q_{\phi}(\mu)\right]=\ln \left(\frac{i c+\lambda-\mu}{i c+\mu-\lambda}\right) .
$$

Дуальными полями называются линейные комбинации

$$
\psi(\lambda)=q_{\psi}(\lambda)+p_{\psi}(\lambda), \quad \phi(\lambda)=q_{\phi}(\lambda)+p_{\phi}(\lambda) .
$$


Очевидно, что в силу (2.6) дуальные поля коммутируют друг с другом:

$$
[\psi(\lambda), \psi(\mu)]=[\phi(\lambda), \phi(\mu)]=[\psi(\lambda), \phi(\mu)]=0 .
$$

С другой стороны, вакуумное среднее от выражений, содержаших дуальные поля, может быть нетривиальным:

$$
S(\lambda, \mu)=-\frac{i c+\lambda-\mu}{i c+\mu-\lambda}=-\left(0\left|e^{\phi(\lambda)} e^{\psi(\mu)}\right| 0\right) .
$$

Таким образом, $S$-матрица может быть записана как вакуумное ожидание от факторизованного выражения вида (2.4). Благодаря свойству (2.8) детерминанты, зависяшие от дуальных полей, хорошо определены. В результате формфакторы могут быть представлены в виде

$$
\langle a|\mathcal{O}(0,0)| b\rangle \sim \operatorname{det} M=(0|\operatorname{det} \widetilde{M}(\phi, \psi)| 0),
$$

где матрица $\widetilde{M}$ является уже локальной, т.е. $\widetilde{M}_{j k}=\widetilde{M}\left(\lambda_{j}, \mu_{k}\right)$.

Аналогичное представление может быть получено и для квадрата модуля формфактора в (2.1), и суммирование по полному набору $\{|b\rangle\}$ может быть проведено под знаком вакуумного среднего. В итоге мы приходим к представлению для среднего (2.1) в терминах вакуумного ожидания от детерминанта некоторой зависяшей от дуальных полей матрицы во вспомогательном фоковском пространстве. В термодинамическом пределе эта матрица переходит в интегральный оператор, и мы получаем

$$
\langle\mathcal{O}(x, t) \mathcal{O}(0,0)\rangle \sim(0|\operatorname{det}(I+V)| 0) .
$$

Здесь ядро оператора $V$ зависит от полей $\phi(\lambda)$ и $\psi(\lambda)$, однако благодаря $(2.8)$ фредгольмов детерминант является хорошо определенным объектом.

Подчеркнем, что здесь описана лишь основная идея метода дуальных полей. В конкретных примерах возможны некоторые модификации, в частности, иногда приходится вводить дополнительные дуальные поля. Подробные формулы можно найти в [1]. Однако основная идея метода всегда состоит в применении факторизации (2.4), которая позволяет свести детерминанты нелокальных матриц к локальным.

Таким образом, корреляционные функции квантового одномерного бозе-газа оказываются пропорциональны вакуумному ожиданию фредгольмова детерминанта, функционально зависящего от дуальных полей. Тем не менее асимптотический анализ данного детерминанта может быть произведен так же, как и для предела свободных фермионов. В силу коммутационных соотношений (2.8) на определенном этапе вычислений можно рассматривать дуальные поля как некоторые классические функции, аналитичные в полосе $|\Im \lambda|<c / 2$. Последнее свойство следует из представления дуальных полей через канонические бозе-поля:

$$
\begin{aligned}
& \phi(\lambda)=\frac{1}{\sqrt{2 \pi}} \int \ln \left(\frac{\frac{i c}{2}+\nu-\lambda}{\frac{i c}{2}-\nu+\lambda}\right)\left(\varphi_{1}^{\dagger}(\nu)+\varphi_{1}(\nu)\right) d \nu, \\
& \psi(\lambda)=\frac{c}{\sqrt{2 \pi}} \int \frac{\varphi_{2}^{\dagger}(\nu)+\varphi_{2}(\nu)}{(\nu-\lambda)^{2}+\frac{c^{2}}{4}} d \nu,
\end{aligned}
$$


где

$$
\left.\left[\varphi_{1}(\lambda), \varphi_{2}^{\dagger}(\mu)\right]=\left[\varphi_{2}(\lambda), \varphi_{1}^{\dagger}(\mu)\right]=\delta(\lambda-\mu), \quad \varphi_{j}(\lambda) \mid 0\right)=0, \quad\left(0 \mid \varphi_{j}^{\dagger}(\lambda)=0 .\right.
$$

Несложно проверить, что коммутационные соотношения между операторами $p_{\psi, \phi}$ и $q_{\psi, \phi}$, определенными по формулам (2.12), в точности совпадают с (2.6).

С помошью методов задачи Римана и классических интегрируемых уравнений можно вычислить асимптотическое поведение детерминанта (2.11) при больших расстояниях и временах (см. [2-5]). Полученное асимптотическое выражение оказывается функционалом от дуальных полей, которые до этого момента рассматривались как некоторые классические функциональные параметры. Для вычисления асимптотики коррелятора теперь необходимо вспомнить о квантовой природе этих операторов и усреднить полученное выражение по вспомогательному вакууму.

Здесь, однако, имеется один важный момент, подробно обсуждавшийся в работе [5]. Дело в том, что операции усреднения и вычисления асимптотики, вообще говоря, неперестановочны. Асимптотика корреляционной функции равна асимптотике вакуумного среднего (2.11), но отнюдь не вакуумному среднему от асимптотики. Именно поэтому результаты работы [11], в которой рассматривались вопросы усреднения некоторых выражений, содержащих дуальные поля, могут быть применены к вычислению корреляционных функций лишь отчасти. Тем не менее в упоминавшейся уже работе [5] было доказано, что существуют асимптотические представления, которые являются устойчивыми по отношению к процедуре усреднения, т.е. асимптотика от вакуумного среднего равна вакуумному среднему от асимптотики. Такое представление для фредгольмова детерминанта было получено в [5], и именно с ним мы будем иметь дело в дальнейшем.

\section{3. ОТОБРАЖЕНИЕ УСРЕДНЕНИЯ}

Асимптотические формулы для фредгольмова детерминанта в терминах дуальных полей, полученные в работе [5], достаточно громоздки (см. (4.1), (4.2) ниже). Поэтому прежде чем заниматься усреднением данных выражений, рассмотрим некоторые более простые и в то же время достаточно общие примеры. Техника, развиваемая в настоящем разделе, позволит нам без особого труда усреднить формулы (4.1), (4.2).

Рассмотрим два дуальных поля $\phi$ и $\psi$, определяемых формулами (2.7). Коммутационные соотношения между операторами рождения и уничтожения зададим в виде

$$
\left[p_{\psi}(\lambda), q_{\phi}(\mu)\right]=\xi(\lambda, \mu), \quad\left[p_{\phi}(\lambda), q_{\psi}(\mu)\right]=\xi(\mu, \lambda),
$$

где $\xi(\lambda, \mu)$ - некоторая функция двух переменных. Здесь нам не нужен явный вид этой функции, однако легко видеть, что, полагая $\xi(\lambda, \mu)=\ln (i c+\mu-\lambda)-\ln (i c+\lambda-\mu)$, мы воспроизводим коммутационные соотношения (2.6). Помимо коммутаторов (3.1) введем еще один дополнительный

$$
\left[p_{\psi}(\lambda), q_{\psi}(\mu)\right]=\eta(\lambda, \mu)=\eta(\mu, \lambda)
$$


где $\eta(\lambda, \mu)$ - симметричная функция двух переменных. Ниже мы увидим, что дополнительное соотношение (3.2) не оказывает сушественного влияния на конечный результат, однако мы пока не будем полагать функцию $\eta(\lambda, \mu)$ равной нулю.

Легко видеть, что основное свойство дуальных полей (2.8) по-прежнему выполняется. Однако, как и ранее, вакуумные ожидания могут быть нетривиальными.

Основной целью данного раздела является вычисление среднего вида

$$
\left(0\left|e^{\mathcal{F}(\psi, \phi)} F(\phi)\right| 0\right)
$$

Здесь $\mathcal{F}(\psi, \phi)$ и $F(\phi)$ суть операторнозначные функционалы (в дальнейшем для краткости просто функционалы) дуальных полей. Функционал $F$ не зависит от поля $\psi$, а $\mathcal{F}$ зависит от этого поля линейно,

$$
\frac{\delta^{2} \mathcal{F}}{\delta \psi^{2}}=0
$$

Все функционалы (и функции) дуальных полей следует понимать в смысле формальных рядов.

Начнем с формулы, полученной в [11],

$$
\left(0\left|\exp \left\{\sum_{k=1}^{N} \alpha \psi\left(\lambda_{k}\right) f_{k}\left(\phi\left(\mu_{k}\right)\right)\right\} F(\phi(\nu))\right| 0\right)=F\left(\sum_{m=1}^{N} z_{m} \xi\left(\lambda_{m}, \nu\right)\right) \frac{E(\eta)}{\operatorname{det} g}
$$

где

$$
E(\eta)=\exp \left\{\frac{1}{2} \sum_{n, m=1}^{N} z_{n} z_{m} \eta\left(\lambda_{n}, \lambda_{m}\right)\right\}
$$

В левой части (3.5) $F(\phi(\nu))$ - функционал (или функция) поля $\phi, \alpha$ - комплексный параметр, функции $f_{k}(z)$ предполагаются голоморфными в окрестности нуля. В правой части числа $z_{j}$ являются корнями системы

$$
z_{j}-\alpha f_{j}\left(\sum_{m=1}^{N} z_{m} \xi\left(\lambda_{m}, \mu_{j}\right)\right)=0
$$

Если данная система имеет несколько решений, то следует выбрать то единственное, которое стремится к нулю при $\alpha \rightarrow 0$ (см. [11]). Наконец, в знаменателе в правой части (3.5) стоит якобиан системы (3.7)

$$
g_{j k}=\frac{\partial}{\partial z_{k}}\left[z_{j}-\alpha f_{j}\left(\sum_{m=1}^{N} z_{m} \xi\left(\lambda_{m}, \mu_{j}\right)\right)\right]
$$

Удобно видоизменить результат (3.5). А именно, введем функцию $w(u)$,

$$
w(u)=\sum_{m=1}^{N} z_{m} \xi\left(\lambda_{m}, u\right)
$$


Умножая каждое из уравнений $(3.7)$ на $\xi\left(\lambda_{j}, u\right)$ и суммируя их, получаем

$$
G(w) \equiv w(u)-\alpha \sum_{m=1}^{N} f_{m}\left(w\left(\mu_{m}\right)\right) \xi\left(\lambda_{m}, u\right)=0 .
$$

Таким образом, вместо системы уравнений (3.7) мы получаем одно уравнение (3.10) для функции $w(u)$, которое, однако, должно выполняться для любого $u$. Корни системы $z_{j}$ выражаются через $w(u)$ по формулам

$$
z_{j}=\alpha f_{j}\left(w\left(\mu_{j}\right)\right)
$$

Легко также видеть, что

$$
\operatorname{det} g=\operatorname{det}\left[\frac{\delta G(w(u))}{\delta w\left(u^{\prime}\right)}\right]
$$

Таким образом, соотношение (3.5) переходит в

$$
\left(0\left|\exp \left\{\sum_{k=1}^{N} \alpha \psi\left(\lambda_{k}\right) f_{k}\left(\phi\left(\mu_{k}\right)\right)\right\} F(\phi(\nu))\right| 0\right)=F(w(\nu)) \frac{E(\eta)}{\operatorname{det} \frac{\delta G}{\delta w}}
$$

Пусть теперь функционал $F(\phi)$ является одновременно функцией некоторого параметра $t: F(\phi)=F(\phi \mid t)$. В асимптотических выражениях для корреляционной функции этот параметр будет играть роль времени. Тогда формула (3.13) может быть записана в виде

$$
\left(0\left|\exp \left\{\sum_{k=1}^{N} \alpha \psi\left(\lambda_{k}\right) f_{k}\left(\phi\left(\mu_{k}\right)\right)\right\} F(\phi(\nu) \mid t)\right| 0\right)=C F(w(\nu) \mid t),
$$

где $C$ - константа, не зависяшая от $t$. При вычислении асимптотики корреляционной функции такого вида постоянные множители нас не будут интересовать. Именно поэтому функцию $\eta(\lambda, \mu)$, дающую вклад только в эту константу, можно положить равной нулю, ибо это никак не влияет на зависимость правой части (3.14) от $t$.

Итак, мы видим, что при усреднении выражений вида (3.5) имеет место отображение оператора $\phi$ в классическую функцию $w$ :

$$
\phi(\nu) \mapsto w(\nu), \quad F(\phi(\nu)) \mapsto F(w(\nu))
$$

где $w(\nu)$ является решением уравнения (3.10). Отображение (3.15) (а также более общее (3.29), см. ниже) будем называть отображением усреднения.

Рассмотрим теперь обобшение функционала

$$
\mathcal{F}(\psi, \phi)=\alpha \sum_{k=1}^{N} \psi\left(\lambda_{k}\right) f_{k}\left(\phi\left(\mu_{k}\right)\right)
$$

являющегося показателем экспоненты в (3.14). Ясно, что в него можно включить производные от полей $\psi^{(m)}(\lambda)$ и $\phi^{(m)}(\lambda)$. Можно рассматривать также непрерывный предел, 
когда сумма по $k$ переходит в интеграл (это было сделано в [11]). Кроме того, вместо конечной суммы по $k$ можно рассматривать бесконечные ряды. Результат вычисления вакуумного среднего во всех перечисленных случаях легко получить соответствующим предельным переходом в формуле (3.14) (или, если необходимы более точные ответы, в $(3.13))$. Важно только, чтобы функционал $\mathcal{F}(\psi, \phi)$ всегда оставался линейным по полю $\psi$ и его производным, в то время как зависимость $\mathcal{F}$ и $F$ от поля $\phi$ может быть весьма сложной.

Таким образом, мы приходим к следуюшей формуле:

$$
\left(0\left|e^{\mathcal{F}(\psi(\cdot), \phi)} F(\phi(\nu) \mid t)\right| 0\right)=C F(w(\nu) \mid t)
$$

где функция $w(u)$ удовлетворяет уравнению

$$
w(u)-\mathcal{F}(\xi(\cdot, u), w)=0
$$

Для функционалов, зависящих от поля $\psi$ нелинейно, вообше говоря, такой простой формулы не существует. Тем не менее в ряде случаев удается обобщить отображение усреднения на более сложные функционалы. Ниже мы рассмотрим пример функционалов, "асимптотически линейных" по полю $\psi(\lambda)$.

Рассмотрим среднее

$$
\left(0\left|e^{\mathcal{F}(\psi(\cdot), \phi, \hat{\Lambda})} F_{c}(\phi(\nu), \hat{\Lambda}) e^{t F_{e}(\phi(\nu), \hat{\Lambda})}\right| 0\right)
$$

Нас будет интересовать поведение этого среднего при $t \rightarrow \infty$. Пусть явная зависимость функционала $\mathcal{F}(\psi(\cdot), \phi, \hat{\Lambda})$ от поля $\psi$ по-прежнему линейна. Кроме того, пусть $\mathcal{F}$ и $F$ зависят от $\psi$ неявным образом через оператор $\hat{\Lambda}$, который определяется как "корень" уравнения

$$
\hat{\Lambda}=\lambda_{0}+\frac{i}{2 t} \psi^{\prime}(\hat{\Lambda}), \quad \hat{\Lambda} \rightarrow \lambda_{0} \quad \text { при } t \rightarrow \infty .
$$

Здесь $\lambda_{0}$ - некоторая константа, штрих означает производную по аргументу. Определение (3.20) является формальным, и его следует понимать в смысле ряда по $t^{-1}$. Если заменить в (3.20) оператор $\psi$ на комплексную функцию, голоморфную в окрестности $\lambda_{0}$, то для любой голоморфной в той же окрестности функции $f(z)$ при соответствующем выборе контура имеем

$$
f(\hat{\Lambda})=\frac{1}{2 \pi i} \oint d z f(z) \frac{1-\frac{i}{2 t} \psi^{\prime \prime}(z)}{z-\lambda_{0}-\frac{i}{2 t} \psi^{\prime}(z)} .
$$

При достаточно больших $t$ знаменатель в (3.21) можно разложить в абсолютно сходяшийся ряд, что дает

$$
f(\hat{\Lambda})=\sum_{n=0}^{\infty} \frac{1}{n !}\left(\frac{i}{2 t}\right)^{n} \frac{d^{n}}{d \lambda_{0}^{n}}\left(1-\frac{i}{2 t} \psi^{\prime \prime}\left(\lambda_{0}\right)\right)\left(\psi^{\prime}\left(\lambda_{0}\right)\right)^{n} f\left(\lambda_{0}\right) .
$$


В частности,

$$
\hat{\Lambda}=\lambda_{0}+\sum_{n=1}^{\infty} \frac{1}{n !}\left(\frac{i}{2 t}\right)^{n} \frac{d^{n-1}}{d \lambda_{0}^{n-1}}\left(\psi^{\prime}\left(\lambda_{0}\right)\right)^{n} .
$$

Мы примем формулу (3.23) в качестве определения оператора $\hat{\Lambda}$. Соответственно функции (и функционалы) от этого оператора следует понимать в смысле (3.22). Очевидно, что при больших $t$ разность между $\lambda_{0}$ и $\hat{\Lambda}$ ведет себя как $t^{-1} \rightarrow 0$. Поэтому мы называем функционал $\mathcal{F}(\psi(\cdot), \phi, \hat{\Lambda})$ асимптотически линейным по $\psi$. Тем не менее замена $\hat{\Lambda}$ на $\lambda_{0}$ в формуле (3.19) незаконна, так как $\psi$ является неограниченным оператором.

Используя (3.23), можно переписать среднее (3.19) в виде

$$
\begin{aligned}
\left(0 \mid \sum_{n=0}^{\infty} \frac{1}{n !}\right. & \left(\frac{i}{2 t}\right)^{n} \frac{\partial^{2 n}}{\partial \lambda_{0}^{n} \partial \beta^{n}} \times \\
& \left.\times\left[e^{\beta \psi^{\prime}\left(\lambda_{0}\right)+\mathcal{F}\left(\psi(\cdot), \phi, \lambda_{0}\right)}\left(1-\frac{i \psi^{\prime \prime}\left(\lambda_{0}\right)}{2 t}\right) F_{c}\left(\phi(\nu), \lambda_{0}\right) e^{t F_{e}\left(\phi(\nu), \lambda_{0}\right)}\right]_{\beta=0} \mid 0\right),
\end{aligned}
$$

что в свою очередь сводится к двойному интегралу

$$
\begin{aligned}
-\frac{2 t}{\pi}\left(0 \mid \int_{0}^{\infty} \rho d \rho e^{2 i(t+i 0) \rho^{2}} \oint \frac{d z}{z} e^{\rho z^{-1} \psi^{\prime}\left(\lambda_{0}+\rho z\right)+\mathcal{F}\left(\psi(\cdot), \phi, \lambda_{0}+\rho z\right)} \times\right. \\
\left.\quad \times\left(1-\frac{i}{2 t} \psi^{\prime \prime}\left(\lambda_{0}+\rho z\right)\right) F_{c}\left(\phi(\nu), \lambda_{0}+\rho z\right) e^{t F_{e}\left(\phi(\nu), \lambda_{0}+\rho z\right)} \mid 0\right),
\end{aligned}
$$

где интегрирование по $z$ ведется по малой окружности вокруг нуля. Напомним, что нас интересует асимптотическое поведение среднего (3.19) при $t \rightarrow \infty$. Несложно проверить, что в этом случае член, содержащий вторую производную $\psi^{\prime \prime}$, дает вклад лишь в постоянный множитель и, следовательно, может быть отброшен. Тогда подынтегральное выражение принимает вид (3.17), и мы можем усреднить его. Имеем

$$
-\frac{2 t}{\pi} \int_{0}^{\infty} \rho d \rho e^{2 i(t+i 0) \rho^{2}} \oint \frac{d z}{z} C(\rho, z, w) e^{t F_{e}\left(w(\nu), \lambda_{0}+\rho z\right)},
$$

где

$$
w(u, \rho, z)-\rho z^{-1} \xi^{\prime}\left(\lambda_{0}+\rho z, u\right)-\mathcal{F}\left(\xi(\cdot, u), w, \lambda_{0}+\rho z\right)=0 .
$$

При $t \rightarrow \infty$ интегралы в (3.24) могут быть оценены по методу стационарной фазы, и мы получим

$$
\left(0\left|e^{\mathcal{F}(\psi(\cdot), \phi, \hat{\Lambda})} F_{c}(\phi(\nu)) e^{t F_{e}(\phi(\nu))}\right| 0\right) \rightarrow C e^{2 i t\left(\Lambda-\lambda_{0}\right) \beta+t F_{e}(w(\nu, \Lambda, \beta), \Lambda)} .
$$

Здесь функция $w(u)$ удовлетворяет уравнению

$$
w(u, \Lambda, \beta)-\beta \xi^{\prime}(\Lambda, u)-\mathcal{F}(\xi(\cdot, u), w, \Lambda)=0,
$$

где числа $\Lambda$ и $\beta$ определяются уравнениями седловой точки:

$$
\begin{aligned}
2 i \beta+\frac{\partial}{\partial \Lambda} F_{e}(w, \Lambda) & =0, \\
2 i\left(\Lambda-\lambda_{0}\right)+\frac{\partial}{\partial \beta} F_{e}(w, \Lambda) & =0 .
\end{aligned}
$$

Итак, в рассмотренном примере мы снова имеем дело с отображением усреднения, однако более обшего вида

$$
\phi(\nu) \mapsto w(\nu), \quad \hat{\Lambda} \mapsto \Lambda, \quad F(\phi, \hat{\Lambda}) \mapsto F(w, \Lambda) .
$$




\section{4. АСИМПТОТИКА КОРРЕЛЯЦИОННОЙ ФУНКЦИИ}

Теперь мы готовы перейти к рассмотрению асимптотики коррелящионной функшии (1.1). В модели квантового одномерного бозе-газа сушествуют две различные фазы, соответствуюшие отрицательному и положительному химическим потенциалам $h$ в гамильтониане (1.2). В то время как при $h<0$ основное состояние совпадает с гольм фоковским вакуумом, при $h>0$ основное состояние представляет собой сферу Ферми. При конечной температуре разница не столь сушественна, но тем не менее знак химического потенциала сказывается на асимптотике корреляционной функции. Соответствуюшие формулы были получены в работе [5]. Приведем явные выражения:

$$
\begin{aligned}
\left\langle\Psi(0,0) \Psi^{\dagger}(x, t)\right\rangle_{T} \longrightarrow & \left(0 \mid C_{-}\left(\phi, \tilde{\phi} \mid \lambda_{0}\right)(2 t)^{-\frac{(\nu(\hat{\Lambda})+1)^{2}}{2}} \exp \left\{\psi(\hat{\Lambda})+i t \hat{\Lambda}^{2}-i x \hat{\Lambda}-i h t\right\} \times\right. \\
\times & \exp \left\{\frac{1}{2 \pi} \int_{-\infty}^{\infty}\left(x-2 \lambda t+i \psi^{\prime}(\lambda)\right) \operatorname{sign}(\hat{\Lambda}-\lambda) \times\right. \\
& \left.\left.\times \ln \left[\frac{e^{\frac{\varepsilon(\lambda)}{T}}-e^{\phi(\lambda) \operatorname{sign}(\lambda-\hat{\Lambda})}}{e^{\frac{\varepsilon(\lambda)}{T}}+1}\right] d \lambda\right\} \mid 0\right)
\end{aligned}
$$

для $h<0$ и

$$
\begin{aligned}
\left\langle\Psi(0,0) \Psi^{\dagger}(x, t)\right\rangle_{T} \longrightarrow & \left(0 \mid C_{+}\left(\phi, \tilde{\phi} \mid \lambda_{0}\right)(2 t)^{-\frac{\nu^{2}(\hat{\Lambda})}{2}} \times\right. \\
\times & \exp \left\{\frac{1}{2} \psi\left(\hat{\Lambda}_{1}\right)+\frac{1}{2} \psi\left(\hat{\Lambda}_{2}\right)+\frac{i t}{2}\left(\hat{\Lambda}_{1}^{2}+\hat{\Lambda}_{2}^{2}\right)-\frac{i x}{2}\left(\hat{\Lambda}_{1}+\hat{\Lambda}_{2}\right)-i h t\right\} \times \\
\times & \exp \left\{\frac{1}{2 \pi} \int_{-\infty}^{\infty}\left(x-2 \lambda t+i \psi^{\prime}(\lambda)\right) \operatorname{sign}(\hat{\Lambda}-\lambda) \times\right. \\
& \left.\left.\times \ln \left[\frac{e^{\frac{\varepsilon(\lambda)}{T}}-e^{\phi(\lambda) \operatorname{sign}(\lambda-\hat{\Lambda})}}{e^{\frac{\varepsilon(\lambda)}{T}}+1} \omega(\lambda)\right] d \lambda\right\} \mid 0\right)
\end{aligned}
$$

для $h>0$.

Сделаем необходимые пояснения. Формулы (4.1), (4.2) получены в предположении, что $t \rightarrow \infty, x \rightarrow \infty$, в то время как отношение $x / 2 t=\lambda_{0}$ остается фиксированным. Функция $\varepsilon(\lambda)$ - решение уравнения Янга-Янга (1.4).

Наиболее важными объектами для нас являются три дуальных поля $\psi(\lambda), \phi(\lambda)$ и $\tilde{\phi}(\lambda)$. Коммутационные соотношения между соответствуюшими операторами рождения и уничтожения суть

$$
\begin{aligned}
{\left[p_{\psi}(\lambda), q_{\phi}(\mu)\right] } & =-\left[p_{\phi}(\lambda), q_{\psi}(\mu)\right]=\xi(\lambda, \mu) \equiv \ln \left(\frac{i c+\mu-\lambda}{i c+\lambda-\mu}\right), \\
{\left[p_{\psi}(\lambda), q_{\psi}(\mu)\right] } & =\left[\tilde{p}_{\phi}(\lambda), q_{\psi}(\mu)\right]= \\
& =\left[p_{\psi}(\lambda), \tilde{q}_{\phi}(\mu)\right]=\eta(\lambda, \mu) \equiv \ln \left(\frac{c^{2}}{(\lambda-\mu)^{2}+c^{2}}\right) .
\end{aligned}
$$

Как обычно, все дуальные поля коммутируют друг с другом. Напомним, что именно данное свойство позволяет на определенном этапе вычислений рассматривать эти 
объекты как классические функции. Формулы (4.1), (4.2) были получены в [5] именно при такой трактовке дуальных полей, хотя некоторые операторные особенности учитывались. В частности, особое внимание уделялось тому, чтобы данные выражения были устойчивы по отношению к процедуре усреднения по вспомогательному вакууму, т.е. чтобы поправки, зависяшие от дуальных полей, оставались малыми и после их усреднения. Присутствие в асимптотических формулах величины $\hat{\Lambda}$ (см. раздел 3 ),

$$
\hat{\Lambda}=\lambda_{0}+\frac{i}{2 t} \psi^{\prime}(\hat{\Lambda})
$$

вызвано именно этой причиной. Все выражения, содержашие оператор $\hat{\Lambda}$, следует понимать в смысле рядов $(3.22),(3.23)$, в частности, интегралы, зависяшие от знаковой функции, по определению равны

$$
\int_{-\infty}^{\infty} f(\lambda, \operatorname{sign}(\hat{\Lambda}-\lambda)) d \lambda=\int_{-\infty}^{\hat{\Lambda}} f(\lambda, 1) d \lambda+\int_{\hat{\Lambda}}^{\infty} f(\lambda,-1) d \lambda .
$$

Для каждого из интегралов в правой части (4.5) можно воспользоваться формулой (3.22). Фактически можно сказать, что $\hat{\Lambda}$ возникает в результате частичного суммирования асимптотического ряда для корреляционной функции.

С операторами $\hat{\Lambda}_{1}$ и $\hat{\Lambda}_{2}$ ситуация аналогичная. Если считать дуальные поля классическими функциями, то $\hat{\Lambda}_{i}$ являются корнями уравнения

$$
\varepsilon\left(\hat{\Lambda}_{i}\right)=T \operatorname{sign}\left(\hat{\Lambda}-\hat{\Lambda}_{i}\right) \phi\left(\hat{\Lambda}_{i}\right), \quad \hat{\Lambda}_{1} \rightarrow-q_{T}, \quad \hat{\Lambda}_{2} \rightarrow q_{T} \quad \text { при } \quad T \rightarrow 0
$$

где $\pm q_{T}-$ нули функции Янга-Янга: $\varepsilon\left( \pm q_{T}\right)=0$. Иными словами, числа $\hat{\Lambda}_{i}$ соответствуют нулям выражения, стояшего под знаком логарифма в интеграле (4.2). В рассматриваемой ситуации, когда $\phi(\lambda)$ - дуальное поле, $\hat{\Lambda}_{i}$ становятся операторами, определяемыми рядами, аналогичными (3.23). Мы потребуем, чтобы при отображении усреднения операторное уравнение (4.6) переходило в обычное уравнение

$$
\varepsilon\left(\Lambda_{i}\right)=T \operatorname{sign}\left(\Lambda-\Lambda_{i}\right) w\left(\Lambda_{i}\right), \quad \Lambda_{1} \rightarrow-q_{T}, \quad \Lambda_{2} \rightarrow q_{T} \quad \text { при } \quad T \rightarrow 0 .
$$

Функция $\omega(\lambda)$, входящая в $(4.2)$, равна

$$
\omega(\lambda)=\operatorname{sign}\left(\lambda-\hat{\Lambda}_{1}\right) \operatorname{sign}\left(\lambda-\hat{\Lambda}_{2}\right) .
$$

Данные знаковые функции следует понимать в том же духе, что и знаковые функции от $\hat{\Lambda}(4.5)$.

Формулы $(4.1),(4.2)$ содержат также функцию $\nu(\hat{\Lambda})$, определяюшую характер степенной зависимости от $t$,

$$
\nu(\hat{\Lambda})=\frac{1}{2 \pi} \ln \left[\left(\frac{e^{\frac{\varepsilon(\hat{\Lambda})}{T}}-e^{-\phi(\hat{\Lambda})}}{e^{\frac{\varepsilon(\hat{\Lambda})}{T}}+1}\right)\left(\frac{e^{\frac{\varepsilon(\hat{\Lambda})}{T}}-e^{\phi(\hat{\Lambda})}}{e^{\frac{\varepsilon(\hat{\Lambda})}{T}}+1}\right)\right] .
$$

5 Теоретическая и математическая физика, т. 121, № 1, 1999 г. 
Явный вид множителей $C_{ \pm}\left(\phi, \tilde{\phi} \mid \lambda_{0}\right)$ в (4.1), (4.2) неизвестен (см. [5]). Известно, однако, что они не зависят от поля $\psi$ и зависят от расстояния $x$ и времени $t$ только через отношение $x / 2 t=\lambda_{0}$, которое, напомним, остается фиксированным.

Несмотря на то что асимптотические формулы (4.1), (4.2) выглядят весьма сложно, они попадают в класс функционалов от дуальных полей, рассмотренных нами в предыдущем разделе. Они асимптотически линейны по полю $\psi(\lambda)$. Операторы $\hat{\Lambda}_{i}$ являются некоторыми неявными функционалами от поля $\phi(\lambda)$. Присутствие третьего поля $\tilde{\phi}(\lambda)$ и наличие неизвестных факторов $C_{ \pm}$не должно нас беспокоить, ибо благодаря тривиальным коммутационным соотношениям

$$
\left[\tilde{p}_{\phi}(\lambda), q_{\phi}(\mu)\right]=\left[p_{\phi}(\lambda), \tilde{q}_{\phi}(\mu)\right]=0
$$

легко видеть, что усреднение поля $\tilde{\phi}$ может дать вклад только в обший постоянньй множитель. Так как нашей задачей является вычисление коррелящионного радиуса, этот множитель несуществен, и мы можем даже положить $\tilde{\phi}=0$.

Итак, применяя отображение усреднения (3.29) к формулам (4.1), (4.2) и пренебрегая как обшим постоянным множителем, так и степенной зависимостью от $t$, имеем

$$
\left\langle\Psi(0,0) \Psi^{\dagger}(x, t)\right\rangle_{T} \longrightarrow e^{-\frac{t}{r_{ \pm}}}
$$

где $r_{ \pm}$соответствуют положительному и отрицательному химическим потенциалам. Мы будем называть $r_{ \pm}$корреляционными радиусами, хотя, строго говоря, они не обязательно вещественны.

При $h<0$

$$
\begin{aligned}
-\frac{t}{r_{-}}= & 2 i t\left(\Lambda-\lambda_{0}\right) \beta+i t \Lambda^{2}-i x \Lambda-i h t+ \\
& +\frac{1}{2 \pi} \int_{-\infty}^{\infty}(x-2 \lambda t) \operatorname{sign}(\Lambda-\lambda) \ln \left[\frac{e^{\frac{\varepsilon(\lambda)}{T}}-e^{w(\lambda) \operatorname{sign}(\lambda-\Lambda)}}{e^{\frac{\varepsilon(\lambda)}{T}}+1}\right] d \lambda .
\end{aligned}
$$

Здесь функция $w(u)$, зависящая от параметров $\Lambda$ и $\beta$, является решением интегрального уравнения

$$
\begin{aligned}
w(u)= & \xi(\Lambda, u)+i \beta K(\Lambda, u)- \\
& -\frac{1}{2 \pi} \int_{-\infty}^{\infty} K(u, \lambda) \operatorname{sign}(\Lambda-\lambda) \ln \left[\frac{e^{\frac{\varepsilon(\lambda)}{T}}-e^{w(\lambda) \operatorname{sign}(\lambda-\Lambda)}}{e^{\frac{\varepsilon(\lambda)}{T}}+1}\right] d \lambda,
\end{aligned}
$$

где

$$
\xi(\lambda, \mu)=\ln \left(\frac{i c+\lambda-\mu}{i c+\mu-\lambda}\right), \quad K(\lambda, \mu)=-i \frac{\partial}{\partial \lambda} \xi(\lambda, \mu)=\frac{2 c}{(\lambda-\mu)^{2}+c^{2}} .
$$


Параметры $\Lambda$ и $\beta$, в свою очередь, определяются из двух дополнительных уравнений

$$
\begin{aligned}
i\left(\Lambda-\lambda_{0}\right) & +\frac{1}{2 \pi} \frac{\partial}{\partial \beta} \int_{-\infty}^{\infty}\left(\lambda_{0}-\lambda\right) \operatorname{sign}(\Lambda-\lambda) \times \\
& \times \ln \left[\frac{e^{\frac{\varepsilon(\lambda)}{T}}-e^{w(\lambda) \operatorname{sign}(\lambda-\Lambda)}}{e^{\frac{\varepsilon(\lambda)}{T}}+1}\right] d \lambda=0, \\
i(\beta+\Lambda- & \left.\lambda_{0}\right)+\frac{1}{2 \pi} \frac{\partial}{\partial \Lambda} \int_{-\infty}^{\infty}\left(\lambda_{0}-\lambda\right) \operatorname{sign}(\Lambda-\lambda) \times \\
& \times \ln \left[\frac{e^{\frac{\varepsilon(\lambda)}{T}}-e^{w(\lambda) \operatorname{sign}(\lambda-\Lambda)}}{e^{\frac{\varepsilon(\lambda)}{T}}+1}\right] d \lambda=0 .
\end{aligned}
$$

Мы также учли, что $x=2 t \lambda_{0}$.

Для положительного химического потенциала формулы похожи, но имеется ряд отличий. Корреляционный радиус дается выражением

$$
\begin{aligned}
-\frac{t}{r_{+}}=2 i t & \left(\Lambda-\lambda_{0}\right) \beta+\frac{i t}{2}\left(\Lambda_{1}^{2}+\Lambda_{2}^{2}-2 h\right)-\frac{i x}{2}\left(\Lambda_{1}+\Lambda_{2}\right)+ \\
+ & \frac{1}{2 \pi} \int_{-\infty}^{\infty}(x-2 \lambda t) \operatorname{sign}(\Lambda-\lambda) \ln \left[\frac{e^{\frac{\varepsilon(\lambda)}{T}}-e^{w(\lambda) \operatorname{sign}(\lambda-\Lambda)}}{e^{\frac{\varepsilon(\lambda)}{T}}+1} \omega(\lambda)\right] d \lambda .
\end{aligned}
$$

При этом функция $w(u)$ удовлетворяет интегральному уравнению

$$
\begin{aligned}
& w(u)=\frac{1}{2}\left(\xi\left(\Lambda_{1}, u\right)+\xi\left(\Lambda_{2}, u\right)\right)+i \beta K(\Lambda, u)- \\
& \quad-\frac{1}{2 \pi} \int_{-\infty}^{\infty} K(u, \lambda) \operatorname{sign}(\Lambda-\lambda) \ln \left[\frac{e^{\frac{\varepsilon(\lambda)}{T}}-e^{w(\lambda) \operatorname{sign}(\lambda-\Lambda)}}{e^{\frac{\varepsilon(\lambda)}{T}}+1} \omega(\lambda)\right] d \lambda,
\end{aligned}
$$

где $\Lambda_{1}$ и $\Lambda_{2}-$ корни уравнения (4.7), и

$$
\omega(\lambda)=\operatorname{sign}\left(\lambda-\Lambda_{1}\right) \operatorname{sign}\left(\lambda-\Lambda_{2}\right) .
$$

Параметры $\Lambda$ и $\beta$ находятся из дополнительных уравнений

$$
\begin{aligned}
& i\left(\Lambda-\lambda_{0}\right)+\frac{1}{2 \pi} \frac{\partial}{\partial \beta} \int_{-\infty}^{\infty}\left(\lambda_{0}-\lambda\right) \operatorname{sign}(\Lambda-\lambda) \times \\
& \times \ln \left[\frac{e^{\frac{\varepsilon(\lambda)}{T}}-e^{w(\lambda) \operatorname{sign}(\lambda-\Lambda)}}{e^{\frac{\varepsilon(\lambda)}{T}}+1} \omega(\lambda)\right] d \lambda=0, \\
& i \beta+\frac{1}{2 \pi} \frac{\partial}{\partial \Lambda} \int_{-\infty}^{\infty}\left(\lambda_{0}-\lambda\right) \operatorname{sign}(\Lambda-\lambda) \times \\
& \times \ln \left[\frac{e^{\frac{\varepsilon(\lambda)}{T}}-e^{w(\lambda) \operatorname{sign}(\lambda-\Lambda)}}{e^{\frac{\varepsilon(\lambda)}{T}}+1} \omega(\lambda)\right] d \lambda=0 .
\end{aligned}
$$


Итак, экспоненциальный закон убывания корреляционной функции (1.1) определяется функцией $w(u)$. Эта функция может быть найдена из системы интегральных уравнений, структура которых похожа на уравнение Янга-Янга (1.4). Поэтому весьма вероятно, что $w(u)$ может быть выражена в терминах наблюдаемых одномерного бозе-газа. В следуюшем разделе мы продемонстрируем это для некоторых предельных случаев.

В заключение этого раздела рассмотрим предел свободных фермионов $c \rightarrow \infty$. Тогда $K(\lambda, \mu)=\xi(\lambda, \mu)=0$ (см. (4.12)) и, следовательно, $w(u)=0$. Кроме того, $\varepsilon(\lambda)=\lambda^{2}-h$, $\Lambda=\lambda_{0}, \beta=0$ и $\Lambda_{1,2}=\mp \sqrt{h}$. Интегральный член в формулах (4.10), (4.15) превращается в

$$
\frac{1}{2 \pi} \int_{-\infty}^{\infty}|x-2 \lambda t| \ln \left|\frac{e^{\frac{\varepsilon(\lambda)}{T}}-1}{e^{\frac{\varepsilon(\lambda)}{T}}+1}\right| d \lambda
$$

В случае положительного химического потенциала данное выражение полностью описывает асимптотическое поведение корреляционной функции, так как $\Lambda_{1}^{2}+\Lambda_{2}^{2}-2 h=0$. При $h<0$ мы имеем добавочный осциллирующий член $-i t\left(\lambda_{0}^{2}+h\right)$. Это в точности соответствует результатам [12].

Отметим, однако, что предел свободных фермионов не является показательным. Действительно, в этом случае все коммутационные соотношения между операторами $p$ и $q(4.3)$ становятся тривиальными, поэтому мы с самого начала могли бы положить в формулах (4.1), (4.2) дуальные поля равными нулю.

\section{5. ПРЕДЕЛЬНЫЕ СЛУЧАИ}

Рассмотрим некоторые предельные случаи для корреляционной функции (1.1). Первый случай соответствует автокорреляции. Хотя мы и рассматривали предел $x \rightarrow \infty$, $t \rightarrow \infty$, но фактически асимптотический анализ, проведенный в [5], основывался на предположении, что отношение $x / 2 t=\lambda_{0}$ конечно. В частности, $\lambda_{0}$ может быть нулем, что и описывает случай $x=0$.

Рассмотрим для определенности случай $h<0$. Несложно проверить, что при $\lambda_{0}=0$ в уравнениях (4.11)-(4.14) можно положить $\Lambda=\beta=0$. Действительно, при нулевых значениях этих параметров из (4.11) следует, что $w(u)$ - нечетная функция. Дифференцируя (4.11) по $\Lambda$ и $\beta$ в нуле, мы находим, что производные $\partial w / \partial \Lambda$ и $\partial w / \partial \beta$ являются четными функциями $u$. Тогда уравнения $(4.13),(4.14)$ выполняются автоматически, и ответ несколько упрошается:

$$
r_{-}^{-1}=i h-\frac{2}{\pi} \int_{0}^{\infty} \lambda \ln \left[\frac{e^{\frac{\varepsilon(\lambda)}{T}}-e^{w(\lambda)}}{e^{\frac{\varepsilon(\lambda)}{T}}+1}\right] d \lambda
$$

где $w(u)$ является решением уравнения

$$
w(u)=-\xi(u, 0)+\frac{1}{2 \pi} \int_{0}^{\infty}(K(\lambda, u)-K(\lambda,-u)) \ln \left[\frac{e^{\frac{\varepsilon(\lambda)}{T}}-e^{w(\lambda)}}{e^{\frac{\varepsilon(\lambda)}{T}}+1}\right] d \lambda
$$


Аналогично для положительного химического потенциала получаем

$$
\begin{aligned}
r_{+}^{-1}= & i\left(h-\Lambda_{1}^{2}\right)-\frac{2}{\pi} \int_{0}^{\infty} \lambda \ln \left[\frac{e^{\frac{\varepsilon(\lambda)}{T}}-e^{w(\lambda)}}{e^{\frac{\varepsilon(\lambda)}{T}}+1} \omega(\lambda)\right] d \lambda \\
w(u)= & \frac{1}{2}\left(\xi\left(\Lambda_{1}, u\right)-\xi\left(\Lambda_{1},-u\right)\right)+ \\
& +\frac{1}{2 \pi} \int_{0}^{\infty}(K(\lambda, u)-K(\lambda,-u)) \ln \left[\frac{e^{\frac{\varepsilon(\lambda)}{T}}-e^{w(\lambda)}}{e^{\frac{\varepsilon(\lambda)}{T}}+1} \omega(\lambda)\right] d \lambda
\end{aligned}
$$

При этом оказывается, что $\Lambda_{2}=-\Lambda_{1}$.

Вторым и наиболее интересным является низкотемпературный предел для случая $h>0$. В этом пределе мы имеем возможность сравнить наши результаты с полученными ранее [7]. Действительно, как уже отмечалось, при $T=0$ асимптотика корреляционной функции должна иметь степенной характер. Это означает, что корреляционный радиус $r_{+} \rightarrow \infty$. Кроме того, при малой температуре коррелящионный радиус был вычислен в линейном приближении в [7], а это значит, что предел малых температур можно рассматривать как хороший тест для наших результатов. Вдобавок мы теперь имеем возможность уточнить результаты [7].

Приведем систему уравнений, описывающих основное состояние для $h>0$ [13]. Прежде всего это уравнение Янга-Янга (1.4), в котором функция $\varepsilon(\lambda)$ имеет два вещественных корня $\varepsilon\left( \pm q_{T}\right)=0$, причем $\varepsilon(\lambda)>0$, если $|\lambda|>q_{T}$, и $\varepsilon(\lambda)<0$ при $|\lambda|<q_{T}$. При $T \rightarrow 0$ корни $\pm q_{T}$ стремятся к некоторым фиксированным значениям (соответствующим гранишам сферы Ферми): $q_{T} \rightarrow q$. Легко видеть, что

$$
\exp \left\{-\frac{\varepsilon(\mu)}{T}\right\} \stackrel{T \rightarrow 0}{\longrightarrow} \begin{cases}0, & |\mu|>q \\ \infty, & |\mu|<q\end{cases}
$$

Таким образом, уравнение (1.4) преврашается в

$$
\varepsilon_{0}(\lambda)=\lambda^{2}-h+\frac{1}{2 \pi} \int_{-q}^{q} K(\lambda, \mu) \varepsilon_{0}(\mu) d \mu, \quad \varepsilon_{0}( \pm q)=0 .
$$

Здесь и в дальнейшем мы будем обозначать индексом 0 наблюдаемые при нулевой температуре. Аналогично уравнения для плотности и фазы рассеяния имеют вид

$$
\begin{aligned}
\rho_{0}(\lambda) & =\frac{1}{2 \pi}+\frac{1}{2 \pi} \int_{-q}^{q} K(\lambda, \mu) \rho_{0}(\mu) d \mu, \\
F_{0}(\lambda, \nu) & =\frac{1}{2 \pi} \int_{-q}^{q} K(\lambda, \mu) F_{0}(\mu, \nu) d \mu+\frac{1}{2 \pi i} \xi(\lambda, \nu) .
\end{aligned}
$$

Производная от импульса частицы и скорость по-прежнему равны

$$
\frac{\partial k_{0}(\lambda)}{\partial \lambda}=2 \pi \rho_{0}(\lambda), \quad v_{0}(\lambda)=\frac{\varepsilon_{0}^{\prime}(\lambda)}{k_{0}^{\prime}(\lambda)}
$$


Приведем еще уравнение для резольвенты интегрального оператора, входящего в формулы (5.5)-(5.7):

$$
R(\lambda, \nu)=\frac{1}{2 \pi} \int_{-q}^{q} K(\lambda, \mu) R(\mu, \nu) d \mu+\frac{1}{2 \pi} K(\lambda, \nu)
$$

Легко видеть, что $R(\lambda, \mu)=-\partial_{\mu} F_{0}(\lambda, \mu)$. Кроме того, из сравнения уравнений (5.6) и (5.7) и с учетом $K(\lambda, \mu)=-i \partial_{\lambda} \xi(\lambda, \mu)$ находим

$$
2 \pi \rho_{0}(\lambda)=1+F_{0}(\lambda,-q)-F_{0}(\lambda, q)
$$

Аналогично уравнению Янга-Янга интегральное уравнение для функции $w(u)$ в пределе $T \rightarrow 0$ также линеаризуется. Пусть, например, $\Lambda_{1}<\Lambda_{2}<\Lambda$. Тогда вместо (4.16) при $T=0$ имеем

$$
w_{0}(u)=\frac{1}{2}(\xi(-q, u)+\xi(q, u))+i \beta K(u, \Lambda)+\frac{1}{2 \pi} \int_{-q}^{q} K(u, \lambda) w_{0}(\lambda) d \lambda .
$$

Здесь мы учли, что $\Lambda_{2}=-\Lambda_{1}=q$ при $T=0$. Сравнивая уравнение $(5.11)$ с уравнениями (5.7) и (5.9), находим

$$
w_{0}(u)=-i \pi\left(F_{0}(u,-q)+F_{0}(u, q)\right)+2 \pi i \beta R(u, \Lambda) .
$$

Условия на числа $\Lambda$ и $\beta$ выглядят следующим образом:

$$
\begin{aligned}
i\left(\Lambda-\lambda_{0}\right)-\frac{1}{2 \pi} \frac{\partial}{\partial \beta} \int_{-q}^{q}\left(\lambda_{0}-\lambda\right) w_{0}(\lambda) d \lambda & =0 \\
i \beta-\frac{1}{2 \pi} \frac{\partial}{\partial \Lambda} \int_{-q}^{q}\left(\lambda_{0}-\lambda\right) w_{0}(\lambda) d \lambda & =0 .
\end{aligned}
$$

Из (5.12) и (5.14) немедленно следует, что $\beta=0$ и, значит,

$$
w_{0}(u)=-i \pi\left(F_{0}(u,-q)+F_{0}(u, q)\right)
$$

Подставляя (5.15) в выражение (4.15) (в котором логарифм также преврашается в линейную функцию), находим

$$
-\frac{t}{r_{+}}=i t\left(q^{2}-h\right)+\frac{i}{2} \int_{-q}^{q}(x-2 \lambda t)\left(F_{0}(\lambda, q)+F_{0}(\lambda,-q)\right) d \lambda .
$$

Легко видеть, что правая часть формулы (5.16) тождественно равна нулю. Действительно, в силу (5.7) интеграл в правой части формулы (5.16) может быть записан в виде

$$
\frac{1}{4 \pi} \int_{-q}^{q}(x-2 \lambda t)(\delta(\lambda-\mu)+R(\lambda, \mu))(\xi(\mu, q)+\xi(\mu,-q)) d \lambda d \mu .
$$


Действуя резольвентой на $(x-2 \lambda t)$ и используя $(5.5)-(5.8)$, получаем

$$
\frac{1}{4 \pi} \int_{-q}^{q}\left(x k_{0}^{\prime}(\lambda)-t \varepsilon_{0}^{\prime}(\lambda)\right)(\xi(\lambda, q)+\xi(\lambda,-q)) d \lambda .
$$

Так как $k_{0}^{\prime}(\lambda)$ является четной функцией, то коэффициент при $x$ равен нулю. После интегрирования по частям имеем

$$
\frac{i t}{4 \pi} \int_{-q}^{q} \varepsilon_{0}(\lambda)(K(\lambda, q)+K(\lambda,-q)) d \lambda
$$

Теперь остается воспользоваться уравнением (5.5) и условием $\varepsilon_{0}( \pm q)=0$, после чего получаем

$$
r_{+}^{-1}=0 \quad \text { при } \quad T=0 .
$$

Таким образом, при нулевой температуре наши формулы действительно дают правильньй результат.

ЗАмЕчАниЕ. Напомним, что введение дуальных полей в некотором смысле эквивалентно факторизации $S$-матрицы. Естественно ожидать, что при усреднении дуальных полей должны возникать объекты, имеющие непосредственное отношение к матрице рассеяния. Однако, в то время как факторизация происходит над голым фоковским вакуумом, усреднение происходит уже после перехода к термодинамическому пределу, т.е. над физическим вакуумом. И замечательно, что при этом вместо “голой” $S$-матрицы возникает функция $w_{0}(u)$, выражаюшаяся через “одетую” фазу рассеяния (5.7) на границах сферы Ферми.

Заметим также, что в приведенных выше вычислениях нам не потребовался явный вид величины $\Lambda$. Несложно, однако, найти ее. Из уравнения (5.13) следует, что

$$
\Lambda-\lambda_{0}-\int_{-q}^{q}\left(\lambda_{0}-\lambda\right) R(\lambda, \Lambda) d \lambda=0
$$

и мы немедленно находим

$$
\lambda_{0} k_{0}^{\prime}(\Lambda)-\frac{1}{2} \varepsilon_{0}^{\prime}(\Lambda)=0, \quad \text { или } \quad v_{0}(\Lambda)=\frac{x}{t} .
$$

Таким образом, величина $\Lambda$ имеет простой физический смысл: частища, имея скорость $v_{0}(\Lambda)$, проходит расстояние $x$ за время $t$.

Для вычисления корреляционного радиуса при малой, но не нулевой температуре необходимы более точные оценки интегралов, содержаших логарифмическую функцию. Пример подобных вычислений приведен в приложении. Ясно, однако, что все низкотемпературные поправки как для корреляционного радиуса, так и в интегральное уравнение на функцию $w(u)$ исходят от окрестностей точек $\pm q$, определяющих границу сферы Ферми. Приведем здесь интегральное уравнение для функции $w(u)$, возникающее в первом порядке по $T$. Будем искать функцию $w(u)$ в виде ряда

$$
w(u)=w_{0}(u)+T w_{1}(u)+T^{2} w_{2}(u)+\cdots,
$$


здесь $w_{0}(u)$ дается формулой $(5.15)$. Для $w_{1}(u)$ имеем

$$
\begin{aligned}
w_{1}(u) & -\frac{1}{2 \pi} \int_{-q}^{q} K(u, \lambda) w_{1}(\lambda) d \lambda= \\
= & -\frac{K(u, q)}{4 \pi \varepsilon_{0}^{\prime}(q)}\left(w_{0}(q)+i \pi\right)^{2}-\frac{K(u,-q)}{4 \pi \varepsilon_{0}^{\prime}(q)}\left(w_{0}(-q)-i \pi\right)^{2}
\end{aligned}
$$

Следовательно,

$$
w_{1}(u)=\frac{\pi^{2}}{2 \varepsilon_{0}^{\prime}(q)}\left(1-F_{0}(q, q)-F_{0}(q,-q)\right)^{2}(R(u, q)+R(u,-q)) .
$$

Здесь учтено, во-первых, что $F_{0}(-\lambda,-\mu)=-F_{0}(\lambda, \mu)$. Во-вторых, мы уже положили параметр $\beta=0$. Легко видеть, что в рамках низкотемпературного приближения это всегда так. Действительно, в любом порядке по $T$ зависимость $w(u)$ от знаковых функций $\operatorname{sign}(\Lambda-u)$ пропадает. Параметры $\Lambda$ и $\beta$ входят в уравнение для $w(u)$ только в виде комбинации $\beta K(\Lambda, u)$ (см. ниже (П.8)). Поэтому производная $\partial w / \partial \Lambda$ всегда оказывается пропорциональной $\beta$, а значит, уравнение (4.18) выполняется при $\beta=0$.

Подставляя $w_{1}(u)$ в выражение для корреляционного радиуса $(4.15)$, после несложных преобразований приходим к формуле

$$
r_{+}^{-1}=\frac{\pi T}{4 v_{0}}\left(1-F_{0}(q, q)-F_{0}(q,-q)\right)^{2}\left(\left|x-v_{0} t\right|+\left|x+v_{0} t\right|\right),
$$

где $v_{0}=v_{0}(q)$. Данный ответ приведен для произвольного расположения корней $\Lambda_{1}$ и $\Lambda_{2}$ относительно точки $\Lambda$. В случае $\Lambda_{1}<\Lambda_{2}<\Lambda$, который мы рассматривали с самого начала, знаки модуля можно опустить.

Сравнивая (5.21) с результатом, полученным в [7],

$$
r_{+}^{-1}=\frac{\pi T}{4 v_{0}} \frac{\left|x-v_{0} t\right|+\left|x+v_{0} t\right|}{\left(2 \pi \rho_{0}(q)\right)^{2}}
$$

мы получаем тождество

$$
\frac{1}{2 \pi \rho_{0}(q)}=1-F_{0}(q, q)-F_{0}(q,-q) .
$$

На первый взгляд такая нелинейная связь между решениями линейного интегрального уравнения выглядит весьма странно. Однако учитывая нелинейное тождество для фазы рассеяния $[14,15]$

$$
\left(1-F_{0}(q, q)\right)^{2}-F_{0}^{2}(q,-q)=1
$$

и связь (5.10), мы убеждаемся в справедливости тождества (5.23). Таким образом, наш ответ для корреляционного радиуса в линейном по температуре приближении в точности совпадает с ответом, полученным в рамках конформной теории поля.

Вычисление высших температурных поправок не встречает серьезных трудностей. Во всех порядках $T^{n}$ уравнения на $w_{n}(u)$ остаются линейными (см. (П.8)), и их решения явно выписываются через резольвенту оператора $I-(1 / 2 \pi) K$ и ее производные. Соответственно корреляционный радиус выражается через функции $k_{0}^{\prime}(q), \varepsilon_{0}^{\prime}(q), F_{0}(q, \pm q)$ и их производные. В частности, поправка к $r_{+}^{-1}$ в порядке $T^{2}$ оказывается нулевой. Таким образом, результат (5.21) справедлив с точностью до членов порядка $T^{3}$. 


\section{ЗАКЛЮЧЕНИЕ}

Мы рассмотрели асимптотическое поведение температурных корреляционных функций квантового одномерного бозе-газа при больших расстояниях и временах и показали, что асимптотика может быть выражена в терминах решений интегральных уравнений, тесно связанных с уравнениями термодинамического анзаца Бете. В низкотемпературном пределе нам удалось найти решения упомянутых уравнений в терминах наблюдаемых данной модели. Мы хотели бы обратить внимание на то, что эти уравнения возникают в процессе усреднения дуальных полей. Если в ранних работах (см., например, [10]) эти квантовые операторы играли вспомогательную роль и в основном использовались для решения некоторых комбинаторных проблем, то теперь полученные результаты позволяют взглянуть на эти объекты с новой точки зрения. В частности, тот факт, что при нулевой температуре решения интегральных уравнений зависят от фазы рассеяния, вряд ли случаен. По-видимому, роль дуальных полей значительно глубже, чем предполагалось ранее.

Благодарности. Автор благодарен А.Р. Итсу и В.Е. Корепину за полезные обсуждения. Настоящая работа была выполнена при поддержке РФФИ (грант № 99-01-000151).

ПРИЛОЖЕНИЕ

В качестве примера низкотемпературного разложения рассмотрим интеграл, входяший в уравнение Янга-Янга,

$$
I=T \int_{-\infty}^{\infty} K(\lambda, \mu) \ln \left(1+e^{-\frac{\varepsilon(\mu)}{T}}\right) d \mu
$$

При $T=0$ вклад в этот интеграл дает, очевидно, только интервал $[-q, q]$ :

$$
I_{T=0}=-\int_{-q}^{q} K(\lambda, \mu) \varepsilon(\mu) d \mu, \quad \varepsilon( \pm q)=0 .
$$

При малой, но конечной температуре поправки к этому выражению возникают за счет вкладов от окрестностей точек $\pm q$. Рассмотрим, например, интеграл

$$
I_{+}=T \int_{q_{T}}^{\infty} K(\lambda, \mu) \ln \left(1+e^{-\frac{\varepsilon(\mu)}{T}}\right) d \mu,
$$

где $\varepsilon\left(q_{T}\right)=0$. Делая замену переменных $\varepsilon(\mu)=T z$, получаем

$$
I_{+}=T^{2} \int_{0}^{\infty} \varphi(\lambda, T z) \ln \left(1+e^{-z}\right) d z
$$

где $\varphi(\lambda, T z)=K(\lambda, \mu) / \varepsilon^{\prime}(\mu)$. Интеграл (П.4) можно разложить в ряд по $T$, имеем

$$
I_{+}=\sum_{n=0}^{\infty} T^{n+2} \varphi^{(n)}(\lambda, 0)\left(1-2^{-n-1}\right) \zeta(n+2),
$$


или в терминах функций $K(\lambda, \mu)$ и $\varepsilon(\mu)$

где

$$
I_{+}=\sum_{n=0}^{\infty} T^{n+2}\left(1-2^{-n-1}\right) \zeta(n+2) D_{q_{T}}^{n} \frac{K\left(\lambda, q_{T}\right)}{\varepsilon^{\prime}\left(q_{T}\right)}
$$

$$
D_{\lambda}=\frac{1}{\varepsilon^{\prime}(\lambda)} \frac{\partial}{\partial \lambda}
$$

Аналогично вычисляются все остальные вклады от окрестностей точек $\pm q_{T}$. Полученные разложения необходимо подставить в уравнение Янга-Янга и положить

$$
\varepsilon(\lambda)=\sum_{n=0}^{\infty} T^{n} \varepsilon_{n}(\lambda), \quad q_{T}=q+\sum_{n=1}^{\infty} T^{n} q_{n} .
$$

Возникаюший бесконечньй набор уравнений можно решать рекуррентным образом. В результате функции $\varepsilon_{n}$ и параметры $q_{n}$ выражаются через $\varepsilon_{0}^{\prime}(q)$ и резольвенту $R(\lambda, \pm q)$.

Аналогично разлагаются в ряд по температуре все интегралы раздела 4 . Приведем полное разложение по температуре интегрального уравнения (4.16) для функции $w(u)$ :

$$
\begin{aligned}
w(u)- & \frac{1}{2 \pi} \int_{-q}^{q} K(u, \lambda) w(\lambda) d \lambda=\frac{1}{2}(\xi(-q, u)+\xi(q, u))+i \beta K(\Lambda, u)- \\
& -\frac{1}{2 \pi} \sum_{\gamma= \pm 1} \sum_{n=2}^{\infty} \frac{(-2 \pi i \gamma)^{n} T^{n-1}}{n !} D_{q_{T}}^{n-2} \times \\
& \times \frac{K\left(u, \gamma q_{T}\right)}{\varepsilon^{\prime}\left(q_{T}\right)}\left[B_{n}\left(1+\gamma \frac{w\left(\gamma q_{T}\right)}{2 \pi i}\right)-B_{n}\left(\frac{1}{2}\right)\right] .
\end{aligned}
$$

Здесь $B_{n}(z)$ - полиномы Бернулли. В данное уравнение необходимо подставить разложения (П.7), после чего функции $w_{n}(u)$ могут быть вычислены в терминах $\varepsilon_{0}^{\prime}(q), \quad R(\lambda, \pm q)$ и их производных.

\section{Список литературы}

[1] T. Kojima, V. E. Korepin, N. A. Slavnov. Commun. Math. Phys. 1997. V. 188. P. 657.

[2] T. Kojima, V. E. Korepin, N. A. Slavnov. Commun. Math. Phys. 1997. V. 189. P. 709.

[3] V. E. Korepin, N. A. Slavnov. J. Phys. A. 1997. V. 30. P. 8241.

[4] Н. А. Славнов. Зап. научн. сем. ПОМИ. 1998. Т. 251. С. 80.

[5] А.Р. Итс, Н. А. Славнов. ТМФ. 1999. Т. 119. № 2. С. 179; math-ph/9811009.

[6] C.N. Yang, C.P. Yang. J. Math. Phys. 1969. V. 10. P. 1115.

[7] A. A. Belavin, A. M. Polyakov, A. B. Zamolodchikov. Nucl. Phys. B. 1984. V. 241. P. 333

[8] V.E. Korepin, N.M. Bogoliubov, A. G. Izergin. Quantum Inverse Scattering Method and Correlation Functions. Cambridge: Cambridge Univ. Press, 1993.

[9] P. Deift, X. Zhou. Ann. of Math. 1995. V. 137. P. 295.

[10] V.E. Korepin. Commun. Math. Phys. 1987. V. 113. P. 177.

[11] V. E. Korepin, N. A. Slavnov. J. Phys. A. 1997. V. 30. P. 8623.

[12] A.R. Its, A. G. Izergin, V. E. Korepin, G. G. Varzugin. Physica D. 1992. V. 54. P. 351.

[13] E. H. Lieb, W. Liniger. Phys. Rev. 1963. V. 130. P. 1605.

[14] V. E. Korepin, N. A. Slavnov. European J. Phys. 1998. V. 5. P. 555; The new identity for the scattering matrix of exactly solvable models, solv-int/9712005.

[15] Н. А. Славнов. ТМФ. 1998. Т. 116. № 3. С. 362. 